OPEN ACCESS

Edited by:

Xiao-Xuan Zhang,

Qingdao Agricultural University, China

Reviewed by:

Huanping Guo,

Army Medical University, China

Qing Liu,

Shanxi Agricultural University, China

*Correspondence:

Zi-Jian Zhao

zhaojaas@163.com

Qing-Feng Meng

mafboy@163.com

Specialty section:

This article was submitted to

Clinical Microbiology,

a section of the journal

Frontiers in Cellular and

Infection Microbiology

Received: 18 August 2021 Accepted: 27 September 2021

Published: 21 October 2021

Citation:

Chen L, Zhao Z-J and Meng Q-F (2021) Detection of Specific lgGAntibodies Against Toxoplasma gondil

in the Serum and Milk of Domestic

Donkeys During Lactation in China: A

Potential Public Health Concern.

Front. Cell. Infect. Microbiol. 11:760400.

doi: 10.3389/fcimb.2021.760400

\section{Detection of Specific IgG-Antibodies Against Toxoplasma gondii in the Serum and Milk of Domestic Donkeys During Lactation in China: A Potential Public Health Concern}

\author{
Long Chen ${ }^{1}$, Zi-Jian Zhao ${ }^{2 *}$ and Qing-Feng Meng ${ }^{3 *}$ \\ 1 Institute of Animal Nutrition and Feed, Jilin Academy of Agricultural Sciences, Gongzhuling, China, 2 Institute of Agro-food \\ Technology, Jilin Academy of Agricultural Sciences, Changchun, China, ${ }^{3}$ Technology Center, Changchun Customs, \\ Changchun, China
}

Toxoplasma gondii is a worldwide zoonotic protozoan. Donkeys are often susceptible to many pathological agents, acting as carriers of pathogens for other animal species and humans. However, data on the prevalence of $T$. gondii in donkeys during lactation and on the status of antibodies against T. gondii in donkey milk are lacking. A cross-sectional study evaluated the variation of the anti-T. gondii antibodies in the blood and milk of domestic donkeys during lactation. A total of 418 domestic donkeys were randomly selected from the Shandong province, eastern China from January 2019 to March 2020. The anti-T. gondii antibodies were found in 11.72\% (49/418) serum and 9.81\% (41/418) milk samples using a commercial ELISA kit, respectively. There was a very high consistency between the serum and milk (Spearman's coefficient $=0.858, p$-value < 0.0001 and Kendall's tau $=0.688, p$-value $<0.0001$ ), particularly at the 45th to 60th day of lactation. The present results of the statistical analysis showed that the history of abortion $(p=0.026$; adjusted $\mathrm{OR}=2.20 ; 95 \% \mathrm{Cl}: 1.15-4.20)$ and cat in the house $(p=0.008$; adjusted $\mathrm{OR}=2.36 ; 95 \% \mathrm{Cl}: 1.26-4.44)$ were significantly associated with $T$. gondii infection in the domestic donkeys. This is the first report to detect antibodies against $T$. gondii in donkey milk in China. These results indicate a potential risk of humans contracting the infection through the consumption of raw milk from the naturally infected donkeys.

Keywords: Toxoplasma gondii, specific IgG-antibodies, domestic donkeys, sera, milk

\section{INTRODUCTION}

Toxoplasmosis is a very important and prevalent foodborne parasitic disease, caused by Toxoplasma gondii, infecting all warm-blooded animals including human beings, livestock, birds, and marine mammals (Dubey, 2010). Normally, T. gondii infection does not result in obvious clinical symptoms. However, the T. gondii infection occurring in pregnant women, organ transplant 
patients, and patients with immune deficiency triggers severe clinical symptoms and even death (Montoya and Liesenfeld, 2004). Thus, T. gondii infection induces huge damages in both the public health sector and the veterinary field. The infection occurs mainly in three ways: congenital transmission, organ transplant/blood transfusion, and through food and water contaminated by either of the three forms of this parasite (tachyzoite, cysts, and oocysts) (Tenter et al., 2000). Usually, raw or undercooked meat, contaminated milk, and unwashed fruit vegetables can induce this parasitic infection (Pinto-Ferreira et al., 2019). To date, no reports suggest evidence of T. gondii infection due to the consumption of donkey's milk, and raw goat's milk has been proven to be associated with the $T$. gondii infection in humans in clinical practice (Camossi et al., 2011).

So far, T. gondii has been reported in the milk of various hosts like a goat (Bezerra et al., 2015; Gazzonis et al., 2019), sheep (Iacobucci et al., 2019), cat (Powell et al., 2001), camel (Saad et al., 2018), buffalo (Dehkordi et al., 2013), cow (Koethe et al., 2017), and even lactating women (Azab et al., 1992). Thus, T. gondii infection is presumed to occur upon the consumption of either of the milk when consumed raw (Boughattas, 2017). Therefore, there is a necessity of identifying the parasitic contamination in donkey's milk (Martini et al., 2014). However, there is limited information available on the prevalence of T. gondii in donkey's milk available worldwide (Haridy et al., 2010; Mancianti et al., 2014; Martini et al., 2014; Perrucci et al., 2021), especially in China, which is one of the world's largest donkey breeding countries.

The consumption of raw milk products has been well-known to pose a very large potential risk, especially in some special groups, such as infants and the aged. Thus, this study aimed to evaluate the prevalence of T. gondii in the serum and milk of domestic donkeys during lactation in China. This would provide primary data regarding the prevalence of $T$. gondii in donkey milk in China and add some new data for the safety of the public.

\section{MATERIALS AND METHODS}

\section{Ethical Statement}

The owners of the donkeys and the local veterinarians were employed to collect the serum and milk from the domestic donkeys. All of the samples were procured with the approval of the owners. All the procedures involving animals were approved by the Animal Care and Ethics Committee of Jilin Academy of Agricultural Sciences.

\section{Sample and Animal Data Collection}

A cross-sectional study was carried out in four donkey culturing cities (Jining, Linyi, Rizhao, and Liaocheng) from the Shandong province, eastern China (Figure 1). A total of 418 serum and 418 milk samples from the domestic donkeys were randomly collected from January 2019 to March 2020. The blood samples and corresponding milk samples were obtained from each of the donkeys. About $10 \mathrm{ml}$ of blood samples was obtained from the jugular vein of the donkeys using the blood lancet and stored in vacuum tubes without anticoagulant agents. Before collecting the milk samples, the teats were firstly disinfected, and then, about $10 \mathrm{ml}$ of milk samples was collected by milking donkeys by humans and stored in sterile tubes. After transferring the samples to the laboratory, the blood samples were centrifuged at $1,500 \mathrm{~g}$ for 10 min and then placed at room temperature for $4 \mathrm{~h}$. Finally, the obtained serum was stored at $-20^{\circ} \mathrm{C}$ until further use. For processing the collected milk samples, the fatty components and the somatic cells were removed according to a previous study (Petruzzelli et al., 2013) and then stored at $-20^{\circ} \mathrm{C}$ until further use. For collecting the animal data, the individual data about the age and history of abortion of each donkey, cats in the house, source of water, and source of fodder were obtained from the owners. Moreover, the day of birth of each donkey was set as day 0 , and the day of lactation was calculated (Gazzonis et al., 2019).

\section{Laboratory Testing for the T. gondii Antibody}

To detect the specific IgG-antibodies against T. gondii in the collected samples, the available commercial ELISA kit (ID Screen ${ }^{\circledR}$ Toxoplasmosis Indirect MultiSpecies, IDVET, Montpellier) was employed according to the instructions of the manufacturer following the protocol described in the previous study (Gazzonis et al., 2018). The absorbance was measured as the optical density (OD) at $450 \mathrm{~nm}$ using a microplate reader (BIO-RAD iMark, United States). The test

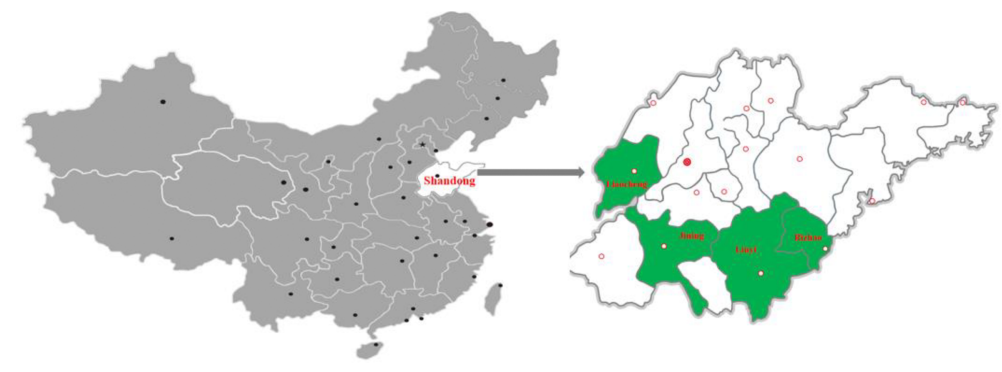

FIGURE 1 | A map of China showing the four cities, Jining, Linyi, Rizhao, and Liaocheng, in Shandong province, eastern China, where the serum and milk samples of the domestic donkeys were collected. 
results were calculated according to the formula provided by the manufacturer:

$$
\begin{aligned}
\mathrm{S} / \mathrm{P} \%= & 100 \times(\mathrm{OD} \text { sample } \\
& - \text { OD negative control }) /(\mathrm{OD} \text { positive control } \\
& - \text { OD negative control })
\end{aligned}
$$

The cutoff value for the positive serum samples and milk samples were set at $\mathrm{S} / \mathrm{P} \% \geq 50 \%$ and $\mathrm{S} / \mathrm{P} \% \geq 21.8 \%$, respectively (Gazzonis et al., 2018).

\section{Statistical Analysis}

The statistical analysis was performed using the SPSS 25.0 software package IBM, (Armonk, NY, United States). p-values less than 0.05 were considered statistically significant. Spearman and Kendall's rank correlation coefficients analyzed the correspondence between sera and milk results. The logistic regression was used to analyze the association between the $T$. gondii infection and potential risk factors. The multivariate logistic analysis was further performed using the full model, including all the potential risk factors in the analyses.

\section{RESULTS}

\section{The T. gondii Antibody Detection in the Serum and Milk Samples}

In total, $11.72 \%(49 / 418)$ serum samples and 9.81\% (41/418) milk samples were found to be positive for the anti-T. gondii antibodies, respectively. Comparing the results obtained from the serum and the milk samples, eight positive serum samples were found to have yielded negative results for the correspondent milk samples, while none of the negative serum samples yielded positive correspondent milk samples.

There was a very high consistency between the results on the serum and milk samples (Spearman's coefficient $=0.858$, $p$-value $<0.0001$ and Kendall's tau $=0.688, p$-value $<0.0001)$. The best agreement was obtained from the 46-60 DP (days from parturition), followed by $0-15 \mathrm{DP}$, while the worst was evident at the second half of the month of lactation (16-30 DP) (Table 1). The trend in the antibody level in the serum and milk was explored: the ELISA S/P\% values of the serum and milk samples were high in the third phase of lactation (31-45 DP) and the fourth phase of lactation (46-60 DP), respectively. Moreover, both the ELISA S/P

\begin{tabular}{|c|c|c|c|c|c|}
\hline \multirow[t]{2}{*}{ Statistical test } & \multicolumn{5}{|c|}{ Days from parturition } \\
\hline & $0-15$ & $16-30$ & $31-45$ & $45-60$ & $>60$ \\
\hline $\begin{array}{l}\text { Kendall's Tau } \\
\text { (p-value) }\end{array}$ & $\begin{array}{c}0.670 \\
(0.000)\end{array}$ & $\begin{array}{c}0.665 \\
(0.000)\end{array}$ & $\begin{array}{c}0.683 \\
(0.000)\end{array}$ & $\begin{array}{c}0.730 \\
(0.000)\end{array}$ & $\begin{array}{r}0.649 \\
(0.000)\end{array}$ \\
\hline $\begin{array}{l}\text { Spearman's coefficient } \\
\text { (p-value) }\end{array}$ & $\begin{array}{c}0.852 \\
(0.000)\end{array}$ & $\begin{array}{c}0.833 \\
(0.000)\end{array}$ & $\begin{array}{c}0.842 \\
(0.000)\end{array}$ & $\begin{array}{c}0.888 \\
(0.000)\end{array}$ & $\begin{array}{c}0.836 \\
(0.000)\end{array}$ \\
\hline
\end{tabular}

TABLE 1 | The conformance between the lactating donkey's serum and milk samples based on the ELISA S/P\% results.
$\%$ values of the serum and milk samples decreased in the last lactation (>60 DP) (Figure 2).

All of the tested domestic donkeys were divided into four age groups. The highest seroprevalence of $T$. gondii in the serum samples was $13.73 \%$ for the age group 37-48 months old, and the highest prevalence of T. gondii in the milk samples was $11.94 \%$ for the age group $>48$ months old (Table 2 ). In terms of region, Linyi (15.31\%) and Rizhao (11.34\%) were found to have the highest prevalence of $T$. gondii in the serum and milk samples, respectively (Table 2). Considering the sampling time, both the highest prevalence of $T$. gondii in the serum and milk samples were found in winter $(16.16 \%$ and 14.14, respectively), and the lowest was found in autumn (8.33\% and $6.82 \%$. respectively) (Table 2). By days from postpartum, the highest prevalence of T. gondii in the serum and milk samples were found in the 46-60 DP group (18.67\%) and the >60 DP group (15.00\%), respectively, but both the lowest prevalence of T. gondii in the serum and milk samples were found in the 0-15 DP group (4.29\% and $2.86 \%$. respectively) (Table 2).

\section{Risk Factors for T. gondii Infection}

In the univariate analysis for the serum samples, two variables were found to be associated with the anti-T. gondii IgG positivity, including the history of abortion ( $p=0.012$; adjusted OR $=2.17$; $95 \%$ CI: 1.18 3.96 ) and cat in the house ( $p=0.002$; adjusted OR $=2.66$; $95 \% \mathrm{CI}$ : $1.45-4.90$ ). Only one variable (cat in the house, $p=0.038$; adjusted $\mathrm{OR}=2.02$; 95\% CI: $1.04-3.91$ ) was found to be associated with the anti-T. gondii IgG positivity in the univariate analysis for the milk samples (Table 2). The following multivariate logistic regression showed that the history of abortion $(p=0.026$; adjusted $\mathrm{OR}=2.20$; 95\% CI: $1.15-4.20)$ and cat in the house $(p=0.008$; adjusted OR $=$ 2.36; 95\% CI: 1.26-4.44) were independent risk factors for T. gondii seropositivity in the domestic donkeys (Table 3).

\section{DISCUSSION}

Donkey's milk has been used since antiquity mainly for its important medicinal properties as well as nutrient values (Li Q. et al., 2020).

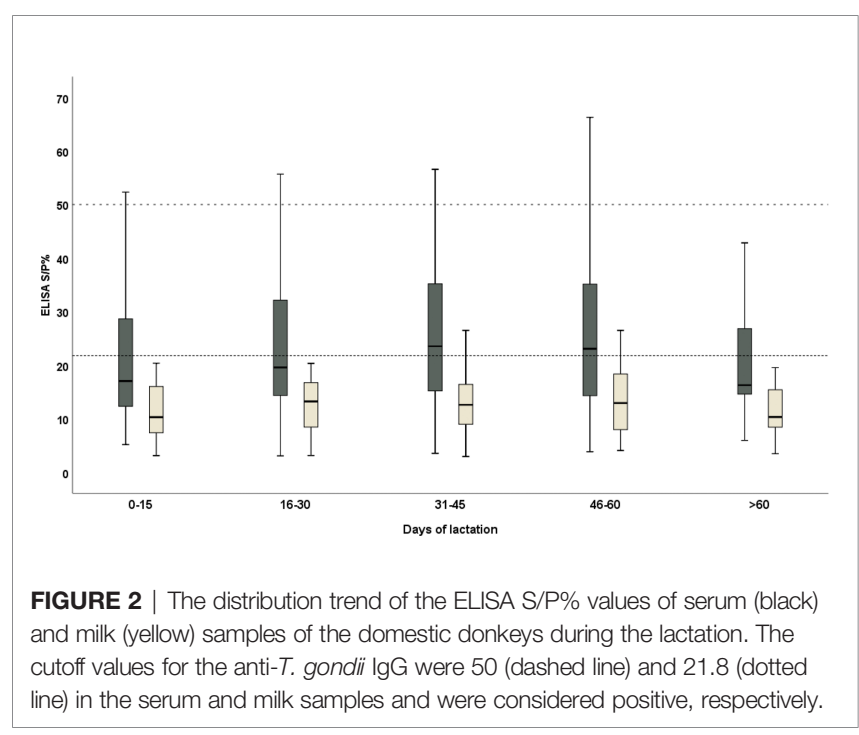


TABLE 2 | Univariate analysis of the variables associated with T. gondii prevalence in the serum and milk samples of the domestic donkeys tested by ELISA.

\begin{tabular}{|c|c|c|c|c|c|c|c|}
\hline \multirow[t]{2}{*}{ Variable } & \multirow[t]{2}{*}{ No. tested } & \multicolumn{3}{|c|}{ Serum } & \multicolumn{3}{|c|}{ Milk } \\
\hline & & Positivity (\%) & $\begin{array}{c}\text { Odds ratio } \\
(95 \% \text { confidence interval) }\end{array}$ & $p$-value & Positivity (\%) & $\begin{array}{c}\text { Odds ratio } \\
(95 \% \text { confidence interval) }\end{array}$ & $p$-value \\
\hline \multicolumn{8}{|l|}{ Age (Months) } \\
\hline$\leqq 24$ & 67 & 4.48 & 0.35 (0.09-1.37) & 0.130 & 4.48 & 0.35 (0.09-1.37) & 0.130 \\
\hline $25-36$ & 182 & 13.19 & $1.12(0.48-2.63)$ & 0.794 & 9.89 & $0.81(0.33-1.96)$ & 0.639 \\
\hline $37-48$ & 102 & 13.73 & $1.17(0.46-2.97)$ & 0.736 & 11.76 & $0.98(0.38-2.55)$ & 0.972 \\
\hline$>48$ & 67 & 11.94 & Reference & & 11.94 & Reference & \\
\hline \multicolumn{8}{|l|}{ Region } \\
\hline Jining & 116 & 9.48 & $0.91(0.38-2.21)$ & 0.842 & 7.76 & $0.82(0.32-2.09)$ & 0.672 \\
\hline Linyi & 98 & 15.31 & $1.58(0.69-3.62)$ & 0.283 & 11.22 & $1.23(0.50-3.03)$ & 0.658 \\
\hline Rizhao & 97 & 12.37 & 1.23 (0.52-2.94) & 0.638 & 11.34 & $1.24(0.50-3.07)$ & 0.640 \\
\hline Liaocheng & 107 & 10.28 & Reference & & 9.35 & Reference & \\
\hline \multicolumn{8}{|l|}{ Sampling time } \\
\hline Spring & 108 & 11.11 & $0.65(0.29-1.45)$ & 0.291 & 7.41 & $0.49(0.19-1.21)$ & 0.122 \\
\hline Summer & 79 & 12.66 & $0.75(0.32-1.76)$ & 0.512 & 12.66 & $0.88(0.37-2.10)$ & 0.774 \\
\hline Autumn & 132 & 8.33 & $0.47(0.21-1.07)$ & 0.071 & 6.82 & $0.44(0.18-1.07)$ & 0.071 \\
\hline Winter & 99 & 16.16 & Reference & & 14.14 & Reference & \\
\hline \multicolumn{8}{|l|}{ History of abortion } \\
\hline Yes & 130 & 17.69 & $2.17(1.18-3.96)$ & $0.012^{*}$ & 12.31 & $1.48(0.76-2.87)$ & 0.251 \\
\hline No & 288 & 9.03 & Reference & & 8.68 & Reference & \\
\hline \multicolumn{8}{|l|}{ Days from postpartum } \\
\hline $0-15$ & 70 & 4.29 & $0.25(0.05-1.37)$ & 0.111 & 2.86 & 0.17 (0.03-1.08) & 0.060 \\
\hline $16-30$ & 164 & 14.02 & $0.92(0.25-3.41)$ & 0.906 & 11.59 & $0.74(0.20-2.77)$ & 0.658 \\
\hline $31-45$ & 89 & 6.74 & $0.41(0.09-1.80)$ & 0.238 & 6.74 & $0.41(0.09-1.80)$ & 0.238 \\
\hline $46-60$ & 75 & 18.67 & $1.30(0.34-5.51)$ & 0.704 & 14.67 & 0.97 (0.24-3.89) & 0.970 \\
\hline$>60$ & 20 & 15.00 & Reference & & 15.00 & Reference & \\
\hline \multicolumn{8}{|l|}{ Cats in house } \\
\hline Yes & 115 & 20.00 & $2.66(1.45-4.90)$ & $0.002^{*}$ & 14.78 & $2.02(1.04-3.91)$ & $0.038^{*}$ \\
\hline No & 303 & 8.58 & Reference & & 7.92 & Reference & \\
\hline \multicolumn{8}{|l|}{ Source of Water } \\
\hline Well & 141 & 10.64 & $0.71(0.35-1.45)$ & 0.344 & 9.22 & 0.78 (0.36-1.69) & 0.781 \\
\hline Tap water & 138 & 10.14 & 0.67 (0.32-1.39) & 0.284 & 8.70 & $0.73(0.33-1.61)$ & 0.438 \\
\hline Well/Tap water & 139 & 14.39 & Reference & & 11.51 & Reference & \\
\hline \multicolumn{8}{|l|}{ Source of fodder } \\
\hline Forage & 96 & 13.54 & $1.17(0.57-2.39)$ & 0.676 & 13.54 & $1.79(0.83-3.85)$ & 0.138 \\
\hline Commercial feed & 111 & 9.91 & $0.82(0.39-1.73)$ & 0.600 & 9.91 & $1.26(0.57-2.78)$ & 0.576 \\
\hline Forage/Commercial feed & 211 & 11.85 & Reference & & 8.06 & Reference & \\
\hline Total & 418 & 11.72 & & & 41 & 9.81 & \\
\hline
\end{tabular}

*Statistically significant.

It is endowed with the potent ability to regulate the immune system to postpone senility, making it a potentially functional health food for inhibiting the progression of some diseases, such as triple-negative breast tumors (Li Q. et al., 2020), type 2 diabetes (Li Y. et al., 2020), and atherosclerosis (Tafaro et al., 2007). Moreover, donkey's milk has been recognized as an ideal alternative to human milk because of its total protein and lactose contents, as well as similar fatty acid and protein profiles (Zhang et al., 2021). Owing to these advantages, there has been a booming global demand for the direct consumption of donkey milk. This escalating demand has to be met by simultaneously and chiefly prioritizing the safety of the consumers, especially considering that many consumers often buy donkey milk directly raw from the farms and individual raisers (Boughattas, 2017). The ingestion of unpasteurized milk has been found to have potential risks and sources of T. gondii infection for children living in rural areas (Radon et al., 2004). Moreover, consumption of unpasteurized milk also elevates the potential risk factor for toxoplasmosis in females with recurrent pregnancy loss (Rehman et al., 2020). The latest China Statistical Yearbook has reported about 2.53 million donkeys in China in 2018 (Luoyizha et al., 2020). Although several studies have been conducted to detect the prevalence of $T$. gondii infection in the donkeys from the different regions of China (Miao et al., 2013; Yang et al., 2013; Zhang et al., 2017; Cong et al., 2018; Meng et al., 2018), the data regarding the prevalence of T. gondii infection in the donkey's milk in China is scarce. This is the first study to estimate the prevalence of the specific IgG-antibodies against $T$. gondii in the milk of the domestic donkeys during lactation in China, which provided important data for controlling and preventing toxoplasmosis in human beings in China.

The present study investigated the anti-T. gondii IgG levels during lactation in the serum and milk samples of the domestic donkeys in China and evaluated the information about the dynamics of specific antibody levels both in the serum and milk. About $9.81 \%$ (41/418) of milk samples were found to be contaminated with T. gondii. Until now, only four studies have 
TABLE 3 | Multivariate logistic regression with a full model for the risk factors of T. gondii infection in the domestic donkeys in China.

\begin{tabular}{lcc}
\hline Variable & $\begin{array}{c}\text { Odds ratio } \\
\text { (95\% confidence } \\
\text { interval) }\end{array}$ & \\
\hline Age (months) ( $(24$ vs. $>48)$ & $0.45(0.11-1.88)$ & 0.274 \\
Age (months) $(25-36$ vs $>48)$ & $1.60(0.64-3.99)$ & 0.316 \\
Age (months) $(37-48$ vs $>48)$ & $1.27(0.48-3.35)$ & 0.633 \\
Region (Jining vs Liaocheng) & $0.83(0.33-2.11)$ & 0.696 \\
Region (Linyi vs. Liaocheng) & $2.06(0.86-4.96)$ & 0.106 \\
Region (Rizhao vs. Liaocheng) & $1.42(0.58-3.47)$ & 0.444 \\
Sampling time (Spring vs. Winter) & $0.69(0.30-1.57)$ & 0.380 \\
Sampling time (Summer vs. Winter) & $1.08(0.43-2.73)$ & 0.866 \\
Sampling time (Autumn vs. Winter) & $0.38(0.17-0.89)$ & 0.026 \\
History of abortion & $2.20(1.15-4.20)$ & 0.017 \\
Days from postpartum (0-15 vs. >60) & $0.17(0.03-0.96)$ & 0.045 \\
Days from postpartum (16-30 vs. >60) & $0.79(0.21-3.01)$ & 0.729 \\
Days from postpartum (31-45 vs. >60) & $0.43(0.09-1.91)$ & 0.265 \\
Days from postpartum (46-60 vs. >60) & $0.99(0.24-4.03)$ & 0.988 \\
Cat in house & $2.36(1.26-4.44)$ & 0.008 \\
Source of Water (Well vs. Well/Tap water) & $0.57(0.27-1.23)$ & 0.152 \\
Source of Water (Tap water vs. Well/Tap water) & $0.62(0.29-1.33)$ & 0.217 \\
Source of fodder (Forage vs. Forage/ & $1.23(0.58-2.61)$ & 0.594 \\
Commercial feed) & & \\
Source of fodder (Commercial feed vs. Forage/ & $0.71(0.33-1.55)$ & 0.391 \\
Commercial feed) & & \\
\hline & &
\end{tabular}

been conducted to explore the contamination status of the milk matrix of donkeys by $T$. gondii globally. In Egypt, the antibodies against $T$. gondii in the milk of a pregnant Egyptian donkey female were detected using an ELISA and reported a contamination rate of $46.3 \%$ (Haridy et al., 2010). In Italy, T. gondii DNA was detected in three of the six tested milk samples using nest-PCR (Mancianti et al., 2014). In another study conducted in Italy, 4 (22.2\%) out of 18 donkeys presented T. gondii DNA in milk (Martini et al., 2014). Simultaneously, the milk quality in the positive donkeys showed a significant difference compared to that in the negative donkeys, suggesting that $T$. gondii infection might induce changes in the milk quality. Moreover, the DNA of T. gondii was found in the milk of three jennies in all the 19 milk samples collected from central Italy by a nest-PCR (Perrucci et al., 2021). In Europe, raw milk collected from any animal can be sold directly to any people (the producer of milk product, a local milk seller, or final consumers) without any processing except refrigeration between 0 and $4^{\circ} \mathrm{C}$ (Mancianti et al., 2014). To sum up, donkey's milk should be considered as a potential pathway of $T$. gondii infection in human beings.

The concordance was explored between the serum and milk collected from the different phases of lactation to find the best agreement in the $45-60$ days from parturition, followed by the first phase ( $0-15$ days from parturition). However, the phase of lactation was not found to be a risk factor influencing the antibody level both in the serum and milk samples in the present study. Unfortunately, there is limited information about the physiological immunoglobulin levels in the donkey's milk during lactation. Based on the present data, in milk, the IgG level demonstrates a little change among the different phases of lactation and the peak was evident in the fourth phase of lactation (46-60 DP). Likewise, in the serum, the IgG level was high in the fourth phase of lactation; subsequently, it decreased sharply in the last phase of lactation (>60 DP).
However, the trends of antibody levels in the milk samples are mostly the same as those in the serum; thus, the IgG trend of milk during lactation might reflect the process of the systemic immunoglobulin production, although more in-depth studies are needed to explain these differences.

As we all know, T. gondii is one of the infectious agents causing early embryonic problems such as abortion, stillbirth, mummification, and death (Dubey, 2009). T. gondii has been considered a potential factor for reproductive failures in domestic animals worldwide (Nayeri et al., 2021). In this study, the domestic donkeys with a history of abortion have been found to demonstrate a significantly higher $T$. gondii seroprevalence compared to those without a history of abortion (Table 2). So, effective control measures and strategies are needed for reducing the rate of abortion in domestic donkeys as well as reducing the economic damage to the livestock industry.

Cats, as the final hosts of this parasite, excrete oocysts via their feces infecting the intermediate hosts such as the domestic animals (Dubey, 2004). The presence of cats in the animals' habitat has been strongly associated with the prevalence of the anti-T. gondii antibodies (Moreira et al., 2019). In this study, the presence of a cat in the house was found to be a significant risk factor for T. gondii seropositivity among these tested domestic donkeys ( $p=0.008$; adjusted OR $=2.36$; $95 \%$ CI: $1.26-4.44$ ) (Table 3). Moreover, the tested domestic donkeys were collected from the rural areas, thus, the number of feral cats may be certainly large. Therefore, it is important to effectively bar cats out of the donkey's habitat to reduce the incidence of infection.

Exploring the transmission route of toxoplasmosis infection in donkeys can provide important suggestions for preventing and treating toxoplasmosis. Undoubtedly, considering the dietary habits of herbivores, they are most likely to contract the infection by ingesting the oocysts that existed in their environment because feline is the final host of $T$. gondii discharging oocysts into the environment. Furthermore, some external forces such as wind, rain, and surface water can facilitate its diffusions in the environment. Although the source of water and source of fodder were not evaluated as the potential risk factors in the present study, these have been identified as the risk factors associated with $T$. gondii infection in domestic animals, such as cow, goat, sheep, and equids (Dubey et al., 2014; Gazzonis et al., 2019; Moreira et al., 2019). Thus, more future studies should be conducted for detecting the T. gondii oocysts in their environment for further assessment of the risk of infection.

In the present study, an available commercial validated ELISA kit was employed to test the serum-milk pairs and an optimal agreement was obtained between the results of the two biological matrices. In this case, it is easier and less expensive to collect the milk samples rather than collecting the serum samples. Moreover, collecting milk is less irritating to the animals. Thus, during the routine disease screening of toxoplasmosis at the individual, herd, and farm levels, this method should be considered for the first round of screening (Schares et al., 2004). However, more studies are needed for supporting the hypothesis of parasite transmission via the ingestion of raw milk or dairy products, including molecular diagnosis and biological methods. 
Although this is the first study detecting the antibodies against $T$. gondii in donkey milk in China, two main limitations cannot be neglected. Firstly, the serum and milk samples were not respectively collected on a different phase of lactation from the same objects. Thus, the concordance between the serum and milk samples may be affected by some objective factors. Secondly, only serological tests were conducted in the present study. The diagnosis of toxoplasmosis merely based on serological tests is ineffective and insufficient. The serological results require a confirmatory diagnostic method that is based on directly demonstrating the parasite in the tissues or biological fluids by tissue culture or mouse inoculation. Thus, more studies should be conducted to verify the current results, including the isolation of the live organisms and more rigorous and standard sampling schemes.

Given the present results, health instruction from the health authorities must be implemented and distributed to the consumers of the animals' milk. Boiling or pasteurization are recommended procedures for eliminating the risk of transmission of $T$. gondii. In addition, more studies should be carried out to evaluate the quantity and viability of $T$. gondii eliminated in the donkey's milk. There is an immense need for some studies based on natural infections, especially in the rural or some individual farmers because they are habituated to consuming raw donkey milk. Both priority and special concerns should be focused on the most vulnerable consumer groups, including the immunocompromised patients, the aged, and babies with milk allergies. Moreover, heat treatment of the milk is strongly recommended before consumption.

\section{DATA AVAILABILITY STATEMENT}

The original contributions presented in the study are included in the article/supplementary material. Further inquiries can be directed to the corresponding authors.

\section{REFERENCES}

Azab, M. E., Kamel, A. M., Makled, K. M., Khattab, H., el-Zayyat, E. A., AboAmer, E. A., et al. (1992). Naturally Occurring Toxoplasma Antibodies in Serum and Milk of Lactating Women. J. Egypt. Soc. Parasitol. 22, 561-568.

Bezerra, M. J., Kim, P. C., Moraes, É.P., Sá, S. G., Albuquerque, P. P., Silva, J. G., et al. (2015). Detection of Toxoplasma gondii in the Milk of Naturally Infected Goats in the Northeast of Brazil. Transbound Emerg. Dis. 62, 421-424. doi: $10.1111 /$ tbed.12160

Boughattas, S. (2017). Toxoplasma Infection and Milk Consumption: MetaAnalysis of Assumptions and Evidences. Crit. Rev. Food. Sci. Nutr. 57, 29242933. doi: 10.1080/10408398.2015.1084993

Camossi, L. G., Greca-Júnior, H., Corrêa, A. P., Richini-Pereira, V. B., Silva, R. C., Da Silva, A. V., et al. (2011). Detection of Toxoplasma gondii DNA in the Milk of Naturally Infected Ewes. Vet. Parasitol. 177, 256-261. doi: 10.1016/ j.vetpar.2010.12.007

Cong, W., Chen, L., Shan, X. F., Qian, A. D., and Meng, Q. F. (2018). First Genetic Characterization of Toxoplasma gondii Infection in Donkey Meat Slaughtered for Human Consumption in Shandong Province, Eastern China. Infect. Genet. Evol. 61, 1-3. doi: 10.1016/j.meegid.2018.03.008

Dehkordi, F. S., Borujeni, M. R., Rahimi, E., and Abdizadeh, R. (2013). Detection of Toxoplasma gondii in Raw Caprine, Ovine, Buffalo, Bovine, and Camel Milk Using Cell Cultivation, Cat Bioassay, Capture ELISA, and PCR Methods in Iran. Foodborne. Pathog. Dis. 10, 120-125. doi: 10.1089/fpd.2012.1311

\section{ETHICS STATEMENT}

All procedures involving animals were approved by the Animal Care and Ethic Committee of Jilin Academy of Agricultural Sciences. Written informed consent was obtained from the owners for the participation of their animals in this study.

\section{AUTHOR CONTRIBUTIONS}

LC: Methodology, formal analysis, and writing-original draft. Z-JZ: Conceptualization, methodology, and writing - review and editing. Q-FM: Conceptualization and writing-review and editing. All authors contributed to the article and approved the submitted version.

\section{FUNDING}

This research was supported by Basic Scientific Research Projects of Jilin Academy of Agricultural Sciences (KYJF2021ZR016); the Funding Program for High-Level Scientific and Technological Innovation Talents introduced by scientific research institutes of Jilin province (project no. 2018001); and the 68th General Grant of China Postdoctoral Science Foundation (project no. 2020M681063).

\section{ACKNOWLEDGMENTS}

The authors are grateful to the owners of donkeys and to local veterinarians for their help in samples collection.

Dubey, J. P. (2004). Toxoplasmosis - A Waterborne Zoonosis. Vet. Parasitol. 126, 57-72. doi: 10.1016/j.vetpar.2004.09.005

Dubey, J. P. (2009). History of the Discovery of the Life Cycle of Toxoplasma gondii. Int. J. Parasitol. 39, 877-82. doi: 10.1016/j.ijpara.2009.01.005

Dubey, J. P. (2010). Toxoplasmosis of Animals and Humans. Boca Raton, FL: CRC Press.

Dubey, J. P., Ness, S. L., Kwok, O. C., Choudhary, S., Mittel, L. D., and Divers, T. J. (2014). Seropositivity of Toxoplasma gondii in Domestic Donkeys (Equus Asinus) and Isolation of T. gondii From Farm Cats. Vet. Parasitol. 199, 18-23. doi: 10.1016/j.vetpar.2013.09.027

Gazzonis, A. L., Zanzani, S. A., Stradiotto, K., Olivieri, E., Villa, L., and Manfredi, M. T. (2018). Toxoplasma gondii Antibodies in Bulk Tank Milk Samples of Caprine Dairy Herds. J. Parasitol. 104, 560-565. doi: 10.1645/17-44

Gazzonis, A. L., Zanzani, S. A., Villa, L., and Manfredi, M. T. (2019). Toxoplasma gondii in Naturally Infected Goats: Monitoring of Specific IgG Levels in Serum and Milk During Lactation and Parasitic DNA Detection in Milk. Prev. Vet. Med. 170, 104738. doi: 10.1016/j.prevetmed.2019.104738

Haridy, F. M., Saleh, N. M., Khalil, H. H., and Morsy, T. A. (2010). AntiToxoplasma gondii Antibodies in Working Donkeys and Donkey's Milk in Greater Cairo, Egypt. J. Egypt. Soc. Parasitol. 40, 459-464.

Iacobucci, E., Taus, N. S., Ueti, M. W., Sukhbaatar, L., Bastsukh, Z., Papageorgiou, S., et al. (2019). Detection and Genotypic Characterization of Toxoplasma gondii DNA Within the Milk of Mongolian Livestock. Parasitol. Res. 118, 2005-2008. doi: 10.1007/s00436-019-06306-w 
Koethe, M., Schade, C., Fehlhaber, K., and Ludewig, M. (2017). Survival of Toxoplasma gondii Tachyzoites in Simulated Gastric Fluid and Cow's Milk. Vet. Parasitol. 233, 111-114. doi: 10.1016/j.vetpar.2016.12.010

Li, Y., Fan, Y., Shaikh, A. S., Wang, Z., Wang, D., and Tan, H. (2020). Dezhou Donkey (Equus Asinus) Milk a Potential Treatment Strategy for Type 2 Diabetes. J. Ethnopharmacol. 246, 112221. doi: 10.1016/j.jep.2019.112221

Li, Q., Li, M., Zhang, J., Shi, X., Yang, M., Zheng, Y., et al. (2020). Donkey Milk Inhibits Triple-Negative Breast Tumor Progression and Is Associated With Increased Cleaved-Caspase-3 Expression. Food. Funct. 11, 3053-3065. doi: 10.1039/C9FO02934F

Luoyizha, W., Wu, X., Zhang, M., Guo, X., Li, H., and Liao, X. (2020). Compared Analysis of Microbial Diversity in Donkey Milk From Xinjiang and Shandong of China Through High-Throughput Sequencing. Food. Res. Int. 2137, 109684. doi: 10.1016/j.foodres.2020.109684

Mancianti, F., Nardoni, S., Papini, R., Mugnaini, L., Martini, M., Altomonte, I., et al. (2014). Detection and Genotyping of Toxoplasma gondii DNA in the Blood and Milk of Naturally Infected Donkeys (Equus Asinus). Parasitol. Vectors. 7, 165. doi: 10.1186/1756-3305-7-165

Martini, M., Altomonte, I., Mancianti, F., Nardoni, S., Mugnaini, L., and Salari, F. (2014). A Preliminary Study on the Quality and Safety of Milk in Donkeys Positive for Toxoplasma gondii. Animal. 8, 1996-1998. doi: 10.1017/ S1751731114001980

Meng, Q. F., Li, D., Yao, G. Z., Zou, Y., Cong, W., and Shan, X. F. (2018). Seroprevalence of Toxoplasma gondii Infection and Variables Associated With Seropositivity in Donkeys in Eastern China. Parasite. 25, 66. doi: 10.1051/ parasite/2018066

Miao, Q., Wang, X., She, L. N., Fan, Y. T., Yuan, F. Z., Yang, J. F., et al. (2013). Seroprevalence of Toxoplasma gondii in Horses and Donkeys in Yunnan Province, Southwestern China. Parasitol. Vectors. 6, 168. doi: 10.1186/17563305-6-168

Montoya, J. G., and Liesenfeld, O. (2004). Toxoplasmosis. Lancet. 363, 1965-1976. doi: 10.1016/S0140-6736(04)16412-X

Moreira, T. R., Sarturi, C., Stelmachtchuk, F. N., Andersson, E., Norlander, E., de Oliveira, F. L. C., et al. (2019). Prevalence of Antibodies Against Toxoplasma gondii and Neospora Spp. In Equids of Western Para, Brazil. Acta Trop. 189, 39-45. doi: 10.1016/j.actatropica.2018.09.023

Nayeri, T., Sarvi, S., Moosazadeh, M., and Daryani, A. (2021). Global Prevalence of Toxoplasma gondii Infection in the Aborted Fetuses and Ruminants That had an Abortion: A Systematic Review and Meta-Analysis. Vet. Parasitol. 290, 109370. doi: 10.1016/j.vetpar.2021.109370

Perrucci, S., Guardone, L., Altomonte, I., Salari, F., Nardoni, S., Martini, M., et al. (2021). Apicomplexan Protozoa Responsible for Reproductive Disorders: Occurrence of DNA in Blood and Milk of Donkeys (Equus Asinus) and Minireview of the Related Literature. Pathogens. 10, 111. doi: 10.3390/ pathogens 10020111

Petruzzelli, A., Amagliani, G., Micci, E., Foglini, M., Di Renzo, E., and Brandi, G.. (2011). Prevalence Assessment of Coxiella burnetii and VerocytotoxinProducing Escherichia coli in Bovine Raw Milk Through Molecular Identification. Food. Control. 32, 532-536. doi: 10.1016/j.foodcont.2013.01.041

Pinto-Ferreira, F., Caldart, E. T., Pasquali, A. K. S., Mitsuka-Breganó, R., Freire, R. L., and Navarro, I. T. (2019). Patterns of Transmission and Sources of Infection in Outbreaks of Human Toxoplasmosis. Emerg. Infect. Dis. 25, 2177-2182. doi: $10.3201 /$ eid2512.181565
Powell, C. C., Brewer, M., and Lappin, M. R. (2001). Detection of Toxoplasma gondii in the Milk of Experimentally Infected Lactating Cats. Vet. Parasitol. 102, 29-33. doi: 10.1016/S0304-4017(01)00521-0

Radon, K., Windstetter, D., Eckart, J., Dressel, H., Leitritz, L., Reichert, J., et al. (2004). Farming Exposure in Childhood, Exposure to Markers of Infections and the Development of Atopy in Rural Subjects. Clin. Exp. Allergy 34, 1178 1183. doi: 10.1111/j.1365-2222.2004.02005.x

Rehman, F., Shah, M., Ali, A., Ahmad, I., Sarwar, M. T., Rapisarda, A. M. C., et al. (2020). Unpasteurised Milk Consumption as a Potential Risk Factor for Toxoplasmosis in Females With Recurrent Pregnancy Loss. J. Obstet. Gynaecol. 40, 1106-1110. doi: 10.1080/01443615.2019.1702630

Saad, N. M., Hussein, A. A. A., and Ewida, R. M. (2018). Occurrence of Toxoplasma gondii in Raw Goat, Sheep, and Camel Milk in Upper Egypt. Vet. World. 11, 1262-1265. doi: 10.14202/vetworld.2018.1262-1265

Schares, G., Bärwald, A., Staubach, C., Wurm, R., Rauser, M., Conraths, F. J., et al. (2004). Adaptation of a Commercial ELISA for the Detection of Antibodies Against Neospora Caninum in Bovine Milk. Vet. Parasitol. 120, 55-63. doi: 10.1016/j.vetpar.2003.11.016

Tafaro, A., Magrone, T., Jirillo, F., Martemucci, G., D'Alessandro, A. G., Amati, L., et al. (2007). Immunological Properties of Donkey's Milk: Its Potential Use in the Prevention of Atherosclerosis. Curr. Pharm. Des. 13, 3711-3717. doi: $10.2174 / 138161207783018590$

Tenter, A. M. M., Heckeroth, A. R. R., and Weiss, L. M. M. (2000). Toxoplasma gondii: From Animals to Humans. Int. J. Parasitol. 30, 1217-1258. doi: 10.1016/S0020-7519(00)00124-7

Yang, N., Mu, M. Y., Yuan, G. M., Zhang, G. X., Li, H. K., and He, J. B. (2013). Seroprevalence of Toxoplasma gondii in Slaughtered Horses and Donkeys in Liaoning Province, Northeastern China. Parasitol. Vectors. 6, 140. doi: 10.1186/ 1756-3305-6-140

Zhang, X., Jiang, B., Ji, C., Li, H., Yang, L., Jiang, G., et al. (2021). Quantitative Label-Free Proteomic Analysis of Milk Fat Globule Membrane in Donkey and Human Milk. Front. Nutr. 8, 670099. doi: 10.3389/fnut.2021.670099

Zhang, X. X., Shi, W., Zhang, N. Z., Shi, K., Li, J. M., Xu, P., et al. (2017). Prevalence and Genetic Characterization of Toxoplasma gondii in Donkeys in Northeastern China. Infect. Genet. Evol. 54, 455-457. doi: 10.1016/ j.meegid.2017.08.008

Conflict of Interest: The authors declare that the research was conducted in the absence of any commercial or financial relationships that could be construed as a potential conflict of interest.

Publisher's Note: All claims expressed in this article are solely those of the authors and do not necessarily represent those of their affiliated organizations, or those of the publisher, the editors and the reviewers. Any product that may be evaluated in this article, or claim that may be made by its manufacturer, is not guaranteed or endorsed by the publisher.

Copyright (c) 2021 Chen, Zhao and Meng. This is an open-access article distributed under the terms of the Creative Commons Attribution License (CC BY). The use, distribution or reproduction in other forums is permitted, provided the original author(s) and the copyright owner(s) are credited and that the original publication in this journal is cited, in accordance with accepted academic practice. No use, distribution or reproduction is permitted which does not comply with these terms. 